\title{
How small are small mutation rates?
}

\author{
Bin Wu • Chaitanya S. Gokhale • Long Wang • \\ Arne Traulsen
}

Received: 21 October 2010 / Revised: 10 May 2011 / Published online: 28 May 2011

(C) Springer-Verlag 2011

\begin{abstract}
We consider evolutionary game dynamics in a finite population of size $N$. When mutations are rare, the population is monomorphic most of the time. Occasionally a mutation arises. It can either reach fixation or go extinct. The evolutionary dynamics of the process under small mutation rates can be approximated by an embedded Markov chain on the pure states. Here we analyze how small the mutation rate should be to make the embedded Markov chain a good approximation by calculating the difference between the real stationary distribution and the approximated one. While for a coexistence game, where the best reply to any strategy is the opposite strategy, it is necessary that the mutation rate $\mu$ is less than $N^{-1 / 2} \exp [-N]$ to ensure that the approximation is good, for all other games, it is sufficient if the mutation rate is smaller than $(N \ln N)^{-1}$. Our results also hold for a wide class of imitation processes under arbitrary selection intensity.
\end{abstract}

Keywords Evolutionary game theory $\cdot$ Mutation rates $\cdot$ Perturbation analysis

Mathematics Subject Classification (2000) 91A22 (Evolutionary games) · 91A40 (Game-theoretic models) · 92D15 (Problems related to evolution)

\footnotetext{
B. Wu · C. S. Gokhale · A. Traulsen ( $\varangle)$

Evolutionary Theory Group, Max-Planck-Institute for Evolutionary Biology,

August-Thienemann-Str. 2, Plön, Germany

e-mail: traulsen@evolbio.mpg.de

B. Wu

e-mail: bin.wu@evolbio.mpg.de

B. Wu $\cdot$ L. Wang

Center for Systems and Control, State Key Laboratory for Turbulence and Complex Systems,

College of Engineering, Peking University, Beijing, China
} 


\section{Introduction}

For evolutionary dynamics in finite populations with mutations, one can think of the evolutionary dynamics on two time scales. In the short run, what is the likelihood that a single mutant or a group of mutants takes over a population? If there is a single $A$ type individual in a population of type $B$, the probability of fixation of $A$ is termed $\phi_{A}$. This quantity has been analytically characterized in population genetics (Crow and Kimura 1970; Karlin and Taylor 1975; Ewens 2004) and has more recently also been applied to evolutionary games (Nowak et al. 2004; Taylor et al. 2004; Fudenberg and Imhof 2006; Nowak 2006; Ohtsuki et al. 2006; Traulsen et al. 2006; Chalub and Souza 2009). On a longer time scale, one can address the average abundance of the available strategies over time (Antal et al. 2009a,b,c; Tarnita et al. 2009). Fudenberg and Imhof (2006), following the work of Foster and Young (1990); Fudenberg and Harris (1992) and Kandori et al. (1993), have developed an approach to deal with this issue. For small mutation rates, the time required for a mutation to occur is much larger than that required for fixation itself. Thus there are at most two strategies in the population simultaneously most of the time. In this case the original stochastic evolutionary process can be approximated by an embedded Markov chain on those states where the population is homogeneous for one strategy. The probability of transition from one homogenous population to another is the corresponding mutation rate multiplied by the fixation probability of the mutant strategy. For simplicity, we assume that all mutation rates are identical.

In particular, when there are only 2 types of strategies, $A$ and $B$, the $2 \times 2$ payoff matrix is given by

$$
\begin{aligned}
& \text { A } B \\
& \begin{array}{l}
A \\
B
\end{array}\left(\begin{array}{ll}
a & b \\
c & d
\end{array}\right),
\end{aligned}
$$

where, $a$ is the payoff of $A$ against $A, b$ is the payoff of $A$ against $B, c$ is the payoff of $B$ against $A$, and finally, $d$ is the payoff of $B$ against $B$. In a well mixed population, an individual interacts with all other individuals with the same probability. A special case would be $b=c$. In this case, one can interpret the game as the interaction of two alleles $A$ and $B$ at a diploid locus (Crow and Kimura 1970; Hofbauer and Sigmund 1998; Bürger 2000; Cressman 1992; van Veelen 2007). Excluding self interactions, the average payoff for each individual of each strategy is given by

$$
\begin{aligned}
\pi_{A}(i) & =a \frac{i-1}{N-1}+b \frac{N-i}{N-1} \quad \text { and } \\
\pi_{B}(i) & =c \frac{i}{N-1}+d \frac{N-i-1}{N-1} .
\end{aligned}
$$

Here, $i$ is the number of individuals playing strategy $A$. Since often the payoff difference is of interest, we substitute $\pi_{A}(i)-\pi_{B}(i)$ by $u i+v$, where $u=\frac{a-b-c+d}{N-1}$ and $v=\frac{N(b-d)-a+d}{N-1}$. 
In this case, the pure population states are 'All play $A$ ' and 'All play $B$ '. The transition probability from 'All play $A$ ' to 'All play $B$ ' is the mutation rate $\mu$ times the fixation probability of strategy $B, \phi_{B}$ (Goel and Richter-Dyn 1974; Nowak 2006). In analogy to this, the transition probability from 'All play $B$ ' to 'All play $A$ ' is the mutation rate $\mu$ times the fixation probability of strategy $A, \phi_{A}$. Thus, the stationary distribution for this Markov chain is

$$
\left(\frac{\phi_{A}}{\phi_{A}+\phi_{B}}, \frac{\phi_{B}}{\phi_{A}+\phi_{B}}\right)
$$

The first element is the average proportion of time spent in state "All play $A$ " while the second element is the average proportion of time spent in state "All play $B$ ". This approach opens up a way to analytically investigate the evolutionary dynamics under mutation, selection and drift provided the mutation rate is sufficiently small (Imhof et al. 2005; Hauert et al. 2007; Van Segbroeck et al. 2009; Wang et al. 2010; Sigmund et al. 2010). However, how small do the mutation rates have to be? Numerical simulations and time scale separation analysis show that $\mu N^{2} \ll 1$ ensures the validity of the approach if the game does not show any stable coexistence (Antal and Scheuring 2006; Hauert et al. 2007; Traulsen et al 2009). However, time scale arguments are often viewed as intuitive tools from physics and are hard to cast into the form of a mathematical proof. Moreover, they do not provide a precise bound for the mutation rate.

Here, by perturbation analysis, we analytically investigate how small the mutation rate must be to make this embedded Markov chain a good approximation of the original one. To this end, we use the total variation distance of probability measures to measure the quality of the approximation of the stationary distribution. For simplicity, we employ the Fermi process (Blume 1993; Szabó and Tóke 1998; Traulsen et al. 2006), a specific yet widely used imitation process. We show that for all games except for the coexistence game, it is sufficient that the mutation rate is smaller than $(N \ln N)^{-1}$ to ensure that the approximation of small mutation rates is good, i.e. $\mu N \ln N \ll 1$. For a coexistence game, however, it is necessary that the mutation rate $\mu$ is less than $N^{-1 / 2} \exp [-N]$. Our result is not only valid for the Fermi process, but also for other imitation processes with continuous derivative of the imitation function (Wu et al. 2010) as well as for the Moran process with different fitness mappings (Traulsen et al 2008; Wu et al. 2010). For any birth death processes with mutations, we also provide a numerically accessible quantity to determine how small the mutation rate should be to make the approximation good.

\section{The Fermi process with mutations}

The Fermi process is a particular birth-death process used to model evolutionary game dynamics in a finite population. In each time step, a random individual is selected. With probability $\mu<1 / 2$, a mutation or exploration event occurs and the focal individual chooses the opposite strategy. With probability $1-\mu$, no mutation occurs. In this case, the focal individual compares its payoff to another randomly chosen individual. If the focal player is playing $A$ and the other plays $B$, then the focal player adopts the 
strategy of the other player with probability

$$
\frac{1}{1+\exp \left[+\beta\left(\pi_{A}(i)-\pi_{B}(i)\right)\right]}
$$

where $\beta$ is the intensity of selection. For small $\beta$, selection is weak and strategy changes occur almost at random. For large $\beta$, only strategies with higher payoff are adopted. Let $i$ be the number of strategy $A$ individuals in the population. Then the transition probabilities from $i$ to $i \pm 1, T_{i}^{ \pm}$, are given by

$$
\begin{aligned}
T_{i}^{+} & =(1-\mu) \frac{i}{N} \frac{N-i}{N} \frac{1}{1+\exp \left[-\beta\left(\pi_{A}(i)-\pi_{B}(i)\right)\right]}+\mu \frac{N-i}{N} \\
T_{i}^{-} & =(1-\mu) \frac{i}{N} \frac{N-i}{N} \frac{1}{1+\exp \left[+\beta\left(\pi_{A}(i)-\pi_{B}(i)\right)\right]}+\mu \frac{i}{N}
\end{aligned}
$$

The probability to stay in state $i$ is $1-T_{i}^{+}-T_{i}^{-}$.

When the mutation rate is nonzero and $\beta$ is finite, this Markov process has no absorbing states. Our birth-death process satisfies the detailed balance condition

$$
\psi_{j-1} T_{j-1}^{+}=\psi_{j} T_{j}^{-} \quad \text { for } 1 \leq j \leq N
$$

where $\psi_{j}$ is the probability that the system is in state $j$ (Kampen 1997; Gardiner 2004; Claussen and Traulsen 2005). The stationary distribution is given by (see Appendix A)

$$
\psi_{j}=\frac{\frac{T_{0}^{+}}{T_{j}^{-}} \prod_{i=1}^{j-1} \frac{T_{i}^{+}}{T_{i}^{-}}}{1+\sum_{k=1}^{N} \frac{T_{0}^{+}}{T_{k}^{-}} \prod_{i=1}^{k-1} \frac{T_{i}^{+}}{T_{i}^{-}}}, \quad 1 \leq j \leq N,
$$

where the empty product is one, $\prod_{i=1}^{0} \frac{T_{i}^{+}}{T_{i}^{-}}=1$. For $j=0$, we have

$$
\psi_{0}=\frac{1}{1+\sum_{k=1}^{N} \frac{T_{0}^{+}}{T_{k}^{-}} \prod_{i=1}^{k-1} \frac{T_{i}^{+}}{T_{i}^{-}}}
$$

For $\mu \rightarrow 0$, we obtain $T_{0}^{+}=\mu=T_{N}^{-} \rightarrow 0$ and thus $\psi_{0} \rightarrow\left(1+\prod_{i=1}^{N-1} \frac{T_{i}^{+}}{T_{i}^{-}}\right)^{-1}$. On the other hand, the numerators of Eq. (8) approach zero for $0<j<N$ due to $\mu \rightarrow 0$. Thus $\psi_{j}$ approach zero as $\mu \rightarrow 0$ for $0<j<N$. Considering the normalization condition, $\sum_{j=0}^{N} \psi_{j}=1$, we have $\psi_{N} \rightarrow 1-\psi_{0}$. Therefore, the ratio between $\psi_{0}$ and $\psi_{N}$ is $\frac{\psi_{0}}{\psi_{N}}=\prod_{i=1}^{N-1} \frac{T_{i}^{+}}{T_{i}^{-}}$. Since $\prod_{i=1}^{N-1} \frac{T_{i}^{+}}{T_{i}^{-}}=\frac{\phi_{B}}{\phi_{A}}$ (Nowak 2006), this recovers Eq. (4). 


\section{Estimating the error in the approximation of the stationary distribution}

For our Markov chain, all possible stationary distributions form a set $S$ denoted by

$$
S=\left\{\left(\psi_{0}, \psi_{1} \cdots \psi_{N}\right) \mid \psi_{i} \geq 0, \sum_{i=0}^{N} \psi_{i}=1\right\}
$$

We follow Durrett (1996) (See also Brémaud 1999; Kallenberg 2002; Levin et al. 2009) to define a measure for the similarity of two such distributions.

Definition Let $z=\left(z_{0}, z_{1} \cdots z_{N}\right)$ and $w=\left(w_{0}, w_{1} \cdots w_{N}\right) \in S$ be two distributions. The total variation distance $d_{T V}(z, w)$ between $v$ and $w$ is defined by

$$
d_{T V}(z, w)=\frac{1}{2} \sum_{i=0}^{N}\left|z_{i}-w_{i}\right|
$$

In particular, two distributions are identical if and only if the total variation distance between them is zero. If they are maximally different, we have $d_{T V}(z, w)=1$. We use this total variation distance as a measure for the quality of the approximation based on the embedded Markov chain described above.

As discussed above, we have from Eqs. (8) and (9)

$$
\begin{aligned}
\lim _{\mu \rightarrow 0} \psi_{0}(\mu) & =\frac{\phi_{B}}{\phi_{A}+\phi_{B}} \\
\lim _{\mu \rightarrow 0} \psi_{i}(\mu) & =0 \text { for } 0<i<N \\
\lim _{\mu \rightarrow 0} \psi_{N}(\mu) & =\frac{\phi_{A}}{\phi_{A}+\phi_{B}}
\end{aligned}
$$

This is consistent with the approach of Fudenberg and Imhof (2006), Eq. (4), which can be viewed as a zeroth order term of an approximation for small mutation rates.

Up to first order, $\psi_{j}(\mu)$ can be approximated by

$$
\psi_{j}(\mu) \approx \psi_{j}(0)+\frac{d}{d \mu} \psi_{j}(0) \mu .
$$

Our goal is to show under which circumstances the second term can be neglected compared to the first one. Based on Eqs. (8) and (9), we can address the derivative in Eq. (13) (See Appendix B.1), which involves the terms

$$
\begin{gathered}
\left.\frac{d}{d \mu} \psi_{0}(\mu)\right|_{\mu=0}=-\left(\psi_{0}(0)\right)^{2}\left(C_{1}+C_{2}\right) \\
\left.\frac{d}{d \mu} \psi_{j}(\mu)\right|_{\mu=0}=\left.\left(\frac{1}{T_{j}^{-}} \prod_{i=1}^{j-1} \frac{T_{i}^{+}}{T_{i}^{-}}\right)\right|_{\mu=0} \psi_{0}(0), \quad 0<j<N,
\end{gathered}
$$


where

$$
\begin{gathered}
C_{1}=\left.\left(\sum_{k=1}^{N-1} \frac{1}{T_{k}^{-}} \prod_{i=1}^{k-1} \frac{T_{i}^{+}}{T_{i}^{-}}\right)\right|_{\mu=0} \\
=\sum_{k=1}^{N-1} \frac{N^{2}\{1+\exp [(u k+v) \beta]\}}{k(N-k)} \exp \left[\left(\frac{u}{2}(k-1)^{2}+\left(\frac{u}{2}+v\right)(k-1)\right) \beta\right]
\end{gathered}
$$

and

$$
\begin{aligned}
C_{2}= & \left.\frac{d}{d \mu}\left(\prod_{i=1}^{N-1} \frac{T_{i}^{+}}{T_{i}^{-}}\right)\right|_{\mu=0} \\
= & N \exp \left[\left(\frac{N-1}{2}(u N+2 v)\right) \beta\right] \exp [-v \beta](1-\exp [(u N+2 v) \beta]) \\
& \times \sum_{i=1}^{N-1} \frac{\exp [-u i \beta]}{i}
\end{aligned}
$$

Here, we have replaced $\pi_{A}(i)-\pi_{B}(i)$ by $u i+v$. The normalization of the distribution, $\sum_{j=0}^{N} \psi_{j}=1$, is determined by the zeroth order term, cf. Eq. (12). Thus, we have $\sum_{j=0}^{N} \frac{d}{d \mu} \psi_{j}=0$, which implies

$$
\left.\frac{d}{d \mu} \psi_{N}(\mu)\right|_{\mu=0}=-\left.\sum_{j=0}^{N-1} \frac{d}{d \mu} \psi_{j}(\mu)\right|_{\mu=0}
$$

We emphasize that Eqs. (14), (15), (16), (18), and (20) are valid for all the birth death processes with mutations. Equations (17), (19) are the special cases obtained by substituting the transition probabilities for the Fermi process, Eqs. (6).

In the following, we denote $\psi(\mu)=\left(\psi_{0}(\mu), \ldots, \psi_{N}(\mu)\right)$ and $\psi(0)=$ $\lim _{\mu \rightarrow 0} \psi(\mu)$. Next, we state our main theorem.

Theorem Assume that the population size $N$ is sufficiently large compared to the product of the selection intensity $\beta$ and the payoff entries in Eq. (1). Evolutionary game dynamics is given by the Fermi process described above. Given an arbitrary $\varepsilon>0$, for all games with $a>c$ or $d>b$ there exists $a$ $\mu^{*}=\varepsilon / G_{1}(N)$, with $G_{1}(N)$ of the order of $N \ln N$, such that if the mutation rate fulfills $\mu<\mu^{*}$, then $d_{T V}(\psi(\mu), \psi(0))<\varepsilon$.

For games with $a<c$ and $d<b$, however, there exists $\mu^{*}=\varepsilon / G_{2}(N)$, where $G_{2}(N)$ is of order $\sqrt{N} \exp [N]$, such that if $d_{T V}(\psi(\mu), \psi(0))<\varepsilon$ then $\mu<\mu^{*}$.

For the proof of this Theorem, we have to infer when the total variation between the distribution with and without mutation is smaller than $\varepsilon$. By Eqs. (11) and (13), 
we have

$$
d_{T V}(\psi(\mu), \psi(0))=\frac{1}{2}\left[\sum_{i=0}^{N}\left|\psi_{i}^{\prime}(0)\right|\right] \mu
$$

Replacing $\psi_{N}^{\prime}(0)$ by Eq. (20) leads to

$$
\begin{aligned}
d_{T V}(\boldsymbol{\psi}(\mu), \boldsymbol{\psi}(0)) & =\frac{1}{2}\left[\sum_{i=0}^{N-1}\left|\psi_{i}^{\prime}(0)\right|+\left|\sum_{i=0}^{N-1} \psi_{i}^{\prime}(0)\right|\right] \mu \\
& \leq \frac{1}{2}\left[\sum_{i=0}^{N-1}\left|\psi_{i}^{\prime}(0)\right|+\sum_{i=0}^{N-1}\left|\psi_{i}^{\prime}(0)\right|\right] \mu \\
& =\sum_{i=0}^{N-1}\left|\psi_{i}^{\prime}(0)\right| \mu
\end{aligned}
$$

First, note that $\psi_{i}^{\prime}(0)>0$ for $i=1, \ldots, N-1$, cf. Eq. (15). Thus, we have

$$
\begin{aligned}
\sum_{i=1}^{N-1}\left|\psi_{i}^{\prime}(0)\right| & =\sum_{i=1}^{N-1} \psi_{i}^{\prime}(0) \\
& =\left.\left(\sum_{i=1}^{N-1} \frac{1}{T_{i}^{-}} \prod_{k=1}^{i-1} \frac{T_{k}^{+}}{T_{k}^{-}}\right)\right|_{\mu=0} \psi_{0}(0) \\
& =C_{1} \psi_{0}(0)
\end{aligned}
$$

On the other hand, we have

$$
\left|\psi_{0}^{\prime}(0)\right|=\left(\psi_{0}(0)\right)^{2}\left|C_{1}+C_{2}\right|
$$

Taking Eqs. (23) and (24) into Expression (22) as well as considering $\psi_{0}(0) \leq 1$ leads to

$$
\begin{aligned}
d_{T V}(\boldsymbol{\psi}(\mu), \boldsymbol{\psi}(0)) & \leq\left(\left|C_{1}+C_{2}\right| \psi_{0}(0)+C_{1}\right) \psi_{0}(0) \mu \\
& \leq\left(\left|C_{1}+C_{2}\right|+C_{1}\right) \mu
\end{aligned}
$$

$C_{1}$ is positive as seen directly from the definition Eq. (17). For $C_{2}$, it is positive when $u N+2 v<0$ and it is negative otherwise. However, for the game fulfilling $u N+2 v>0$, we can look at a transformed game in which $A$ and $B$ are exchanged. This leads to $\tilde{u}=u$ and $\tilde{v}=(N(c-a)-d+a) /(N-1)$. Using $v+\tilde{v}=-\tilde{u} N$ leads to $\tilde{u} N+2 \tilde{v}<0$. Since the exchange of strategies does not affect our general result, we thus always consider the game satisfying $u N+2 v<0$. In this case, both $C_{1}$ and $C_{2}$ are positive, yielding

$$
d_{T V}(\psi(\mu), \psi(0)) \leq\left(2 C_{1}+C_{2}\right) \mu
$$


Thus, the scaling of $2 C_{1}+C_{2}$ with $N$ allows us to assess how the total variation distance scales with $N$. For games except for the coexistence game, i.e. for $a>c$ or for $b<d$ in Eq. (1), we can derive an upper bound for the mutation rate: $2 C_{1}+C_{2}$ is smaller than a quantity $G_{1}(N)$ of order $N \ln N$ for large $N$ (See Appendices B.2.1 and B.2.2). Hence, we have $d_{T V}(\boldsymbol{\psi}(\mu), \boldsymbol{\psi}(0)) \leq G_{1}(N) \mu$. For any $\varepsilon>0$, we define $u^{*}=\varepsilon / G_{1}(N)$ and whenever $u<u^{*}$, the error we are making when considering the stationary distribution without mutations instead of the one with mutations is smaller than $\varepsilon, d_{T V}(\psi(\mu), \psi(0))<\varepsilon$. Since we can specify an upper bound for $u^{*}$, the condition is sufficient.

For the coexistence game ( $a<c$ and $b>d$ ), we only find a lower bound: $2 C_{1}+C_{2}$ is greater than a quantity $G_{2}(N)$ of order $\sqrt{N} \exp [N]$ for large $N$ (See Appendix B.2.3). For any $\varepsilon>0$, we can define $u^{*}=\varepsilon / G_{2}(N)$. Only when $u<u^{*}$, the error of our approximation can be small, $d_{T V}(\psi(\mu), \psi(0))<\varepsilon$.

This completes the proof of our Theorem.

Corollary 1 As per the above Theorem, for games with $a>c$ or $d>b$, i.e. $2 \times 2$ games except for the coexistence game, if $\mu$ is smaller than the error $\varepsilon$ times $(N \ln N)^{-1}$, then $d_{T V}(\psi(\mu), \psi(0))<\varepsilon$. Thus $\mu<\varepsilon(N \ln N)^{-1}$ is a sufficient condition to ensure that the embedded Markov chain is a good approximation of the original one (See Fig. 1). In analogy to this, for coexistence game, the Theorem implies that $\mu<\varepsilon \exp [-N] N^{-1 / 2}$ is only a necessary condition.

In the following we investigate what the mutation rate should be for neutral evolution, $\beta=0$. In this case, the selection is absent and the strategies evolve due to mutation and neutral drift. Equation (24) still holds since we do not employ $\beta$ to obtain Eq. (24). In this case, we have $C_{2}=0$ and

$$
\begin{aligned}
C_{1} & =\sum_{i=1}^{N-1} \frac{2 N^{2}}{i(N-i)} \\
& =2 N \sum_{i=1}^{N-1}\left(\frac{1}{i}+\frac{1}{N-i}\right) \\
& =4 N H_{N-1}
\end{aligned}
$$

where $H_{N-1}=\sum_{i=1}^{N-1} 1 / i$ is the Harmonic number, which is of order $\ln N$ for large $N$. Thus, for $\beta=0$, we have $2 C_{1}+C_{2}=8 N H_{N-1}$, which is of the order of $N \ln N$. From Eq. (26), we conclude that in this special case of neutral selection, a mutation rate of the order of $(N \ln N)^{-1}$ is sufficient to make the approximation good.

Finally, we address the validity of our approach for other processes. The Fermi process is a special imitation process whose imitation function is the Fermi function, Eq. (5). In general, any meaningful imitation process must have an increasing imitation function (Wu et al. 2010). Here, in addition we require differentiability, which is not fulfilled for all such processes (Santos and Pacheco 2005; Szabó and Fáth 2007; Roca et al. 2009). For a general imitation function, which is increasing and differentiable, we show the Theorem is also valid, provided the first order derivative of the imitation function is continuous (See Appendix C). 

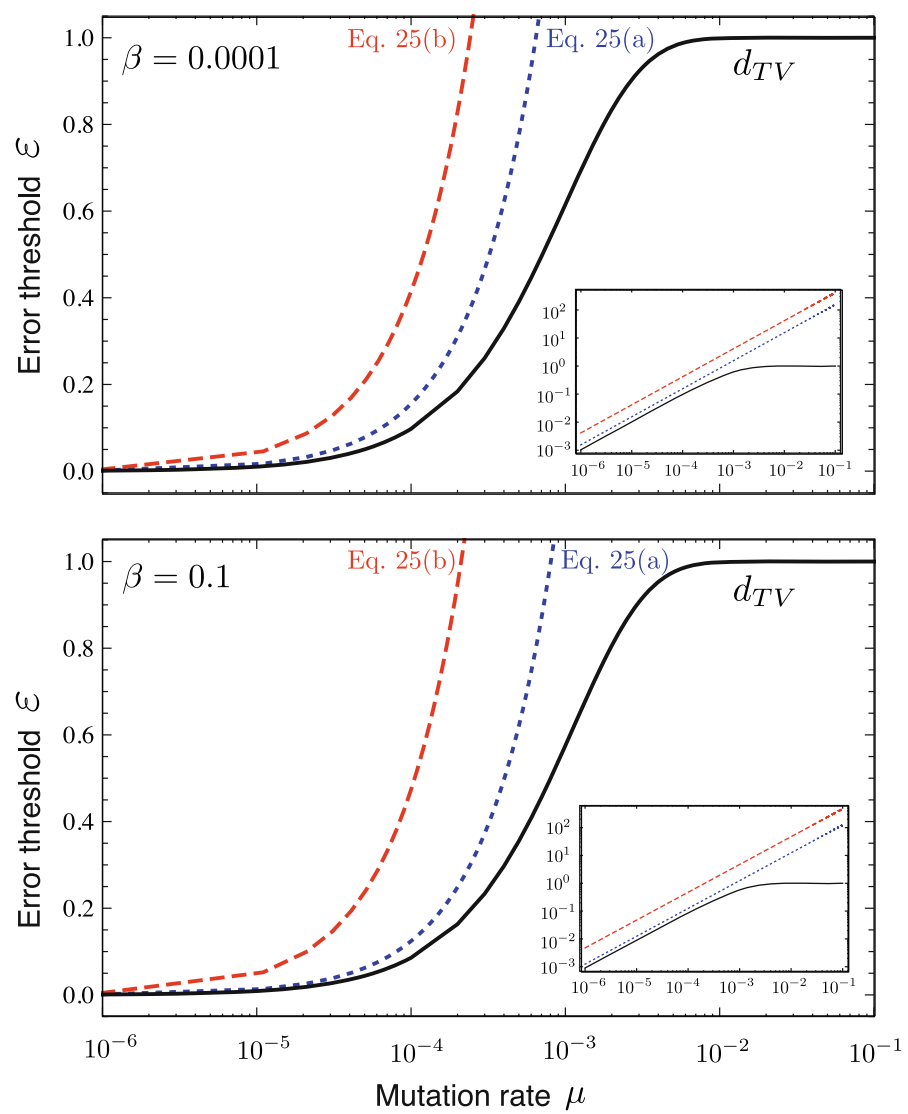

Fig. 1 Total variation distance $d_{T V}$ between the real stationary distribution and the approximation based on small mutation rates. The plots show the exact total variation distance $d_{T V}$ as well as two approximation of $d_{T V}$. The exact total variation distance is calculated for a co-ordination game with $a=1.2, b=$ $1.0, c=1.0$ and $d=1.1$ and plotted as $d_{T V}$ (thick curve) given by Eq. (11). The stationary distributions were calculated from Eqs. (8) and (9). The dotted line is the right hand side of Eq. (25a) namely, $\left(\left|C_{1}+C_{2}\right| \psi_{0}(0)+C_{1}\right) \psi_{0}(0) \mu$. The dashed line is the right hand side of the inequality in Eq. (25b) which approximates the dotted line by $\left(\left|C_{1}+C_{2}\right|+C_{1}\right) \mu$. The numerical analysis was performed for a population of size 100 , for different values of the selection intensity $\beta$. As the selection intensity increases the approximations deviates further from the exact result. The deviation is a quantitative distortion and not qualitative as can be seen from the inset $\log -\log$ plots

\section{Discussion and conclusion}

We have investigated how small the mutation rate should be to make the stationary distribution obtained with a mutation rate going to zero a good approximation of the "real" stochastic process with nonzero mutation rate. For a non-coexistence game, it is sufficient that the mutation rate is smaller than a quantity of the order of $(N \ln N)^{-1}$. For a coexistence game, however, it is necessary that the mutation rate $\mu$ is less than a quantity of the order of $N^{-1 / 2} \exp [-N]$. These results are valid for any nonzero selection intensity. When the selection intensity vanishes, the mutation rate $\mu$ of order 
$(N \ln N)^{-1}$ is sufficient to make the approximation good. Therefore, we can say that the order of $\mu$, which makes the approximation good, does not change compared to the neutral case provided the game allows no coexistence.

In population genetics, the effective selection intensity $N \beta$ plays an important role. Our result is based on a fixed selection intensity $\beta$ and a large, but finite population size $N$. Diffusion approximation is based on a large population size, such that the discrete process is approximated by a continuous one (Ewens 2004; Sella and Hirsh 2005). Typically, this requires a rescaling of $\beta$ in the limit of $N \rightarrow \infty$, such that $N \beta$ remains constant. If we would assume that the effective selection intensity $N \beta$ is of order 1 , we would find that the mutation bound is of order $1 /(N \ln N)$ for all games. In fact, all through the manuscript, we implicitly rescale $\beta=1$. Here, we rewrite the critical quantity $2 C_{1}+C_{2}$ as a function of $\beta, 2 C_{1}(\beta)+C_{2}(\beta)=2 C_{1}(N \beta / N)+C_{2}(N \beta / N)$. If $N \beta$ is of order 1 , it is equivalent to consider the order of $2 C_{1}(1 / N)+C_{2}(1 / N)$. Similar to the calculations in the appendix, we would find that $2 C_{1}(1 / N)+C_{2}(1 / N)$ is of $\operatorname{order} N \ln (N)$ for all games.

Antal and Scheuring (2006) have shown that the conditional fixation time for noncoexistence games is of the order of $N \ln N$. This provides the basis for a procedure called time scale separation: Given that the time to fixation is much longer than the time between two mutations, the system can be approximated by an embedded Markov chain on the monomorphic states and in this way the average abundance can be calculated. Based on the result of Antal and Scheuring (2006), this requires $\mu \ll(N \ln N)^{-1}$ for non-coexistence games. This intuitive and powerful reasoning has been applied in several models (Imhof and Nowak 2006; Hauert et al. 2007; Traulsen and Nowak 2007; Sigmund et al. 2010).

While our results lead to the same scaling with $N$, it allows to make a more concrete statement on the quality of the approximation. Instead of the somewhat intuitive notion of "a good approximation" and "much smaller than", we can now specify a numerical error bound in the total variation distance, which leads to a numerical value for the maximal mutation rate.

To formulate the problem mathematically, we have introduced the total variation distance to measure how "good" the embedded Markov chain is compared to the original one. We can also introduce other measures of distances. A natural question arises: How much does the definition of the distance influence the results? In analogy to Eq. (11), the distance between $z$ and $w$ induced by the $p-$ Norm is given by

$$
\|z-w\|_{p}=\left(\sum_{i=0}^{N}\left|z_{i}-w_{i}\right|^{p}\right)^{\frac{1}{p}}, \quad p \geq 1
$$

In particular, we have $\|\boldsymbol{\psi}(\mu)-\boldsymbol{\psi}(0)\|_{1}=2 d_{T V}(\boldsymbol{\psi}(\mu), \boldsymbol{\psi}(0))$ by the definition of the total variation distance. Since $\|\psi(\mu)-\psi(0)\|_{p} \leq\|\boldsymbol{\psi}(\mu)-\boldsymbol{\psi}(0)\|_{1}$ for $p>1$ as well as $d_{T V}(\psi(\mu), \psi(0)) \leq G_{1}(N) \mu$ for a non-coexistence game, we have $\|\boldsymbol{\psi}(\mu)-\psi(0)\|_{p} \leq 2 G_{1}(N) \mu$ for $p>1$. By identical arguments, the Theorem is also valid for non-coexistence games under this definition of distance. For a coexistence game, however, we have $\|\psi(\mu)-\psi(0)\|_{p} \geq$ 
$\|\boldsymbol{\psi}(\mu)-\boldsymbol{\psi}(0)\|_{1} /(N+1)$ for $p>1$. In analogy to the above discussion, the Theorem should be reformed by replacing $G_{2}(N)=\sqrt{N} \exp [N]$ by $\exp [N] / \sqrt{N}$. Therefore, our Theorem is robust with respect to the definition of distance for a non-coexistence game while needs reformation for a coexistence game. But the reformed theory illustrates that the critical mutation rate for a coexistence game decreases more rapidly compared to that of the non-coexistence games. Thus the results are qualitatively robust with respect to the definition of the distance.

In addition, our result based on the total variation distance is consistent with an intuitive measure in population genetics, the probability that a population is polymorphic. On one hand, the polymorphic states are $i=1, \ldots, N-1$, thus the probability that a population is polymorphic is $F_{1}=\sum_{i=1}^{N-1} \psi_{i}(\mu) \approx \sum_{i=1}^{N-1} \psi_{i}^{\prime}(0) \mu$ by Eqs. (12) and (13). The order of $\sum_{i=1}^{N-1} \psi_{i}^{\prime}(0)$ is identical with that of $C_{1} \psi_{0}(0)$ (see Appendix B). On the other hand, the total variation distance is estimated by $F_{2}=\psi_{0}^{\prime}(0) \mu+F_{1}$ by Eq. (25a). The order of $\psi_{0}^{\prime}(0)$ is identical with that of order $\psi_{0}^{2}(0) C_{1}$, which is lower than that of $\psi_{0}(0) C_{1}$. Hence $F_{1}$ and $F_{2}$ are identical in the order with large population size $N$. Thus the Theorem is robust under this measure.

We have shown that the Theorem is not only valid for the Fermi process, but also for a general imitation process with continuous derivative of the imitation function (Wu et al. 2010). By definition an imitation process involves an imitator and a role model and the strategy of the role model can be adopted by the imitator. Individuals are more likely to imitate those with higher fitness. This has been termed as 'monotonicity' by Fudenberg and Imhof (2008). In addition, the Theorem is also valid for the Moran process with continuous differentiable fitness mappings. The proof is quite similar to that of the general imitation process and thus we do not show it in the appendix. For the Moran process, the monotonicity of the payoff to fitness mapping is also needed. This ensures that individuals with higher payoff have more chance to reproduce.

Since the proof of the Theorem depends only on $C_{1}$ and $C_{2}$ as defined in Eqs. (16) and (18) and the triangle inequality used in Eqs. (22), (25a) is valid for general evolutionary processes that can be described by a birth death process with mutations. The Moran processes with different fitness functions are of this kind (Wu et al. 2010). Therefore, for any such process, given the error bound $\varepsilon$, the critical mutation bound that ensures that the approximation by the embedded Markov chain is good, i.e., $d_{T V}(\psi(\mu), \psi(0)) \leq \varepsilon$, is $\varepsilon /\left(\left|C_{1}+C_{2}\right|+C_{1}\right)$. In other words, the numerical value of $\left|C_{1}+C_{2}\right|+C_{1}$ is sufficient to determine the critical mutation bound. Considering that $\left|C_{1}+C_{2}\right|+C_{1}$ is numerically accessible, it paves the way to determine the critical mutation bound. This mutation bound for the Fermi process is given in Appendix B.3

In contrast to $2 \times 2$ games, it would be challenging to address what the mutation rate has to be for more than two strategies. For multi-strategy games it is difficult to obtain the exact stationary distribution. However, when there are at most two strategies in the population, then pairwise competition between all strategies is the main force of selection, therefore, our results for $2 \times 2$ can still shed light on how small the mutation rate should be. In fact, for $n \times n$ games, we optimistically speculate that our Theorem is also valid, whenever there are no stable internal equilibria in the simplex and the sub-simplices. 
Acknowledgments We thank Drew Fudenberg for initiating this work and Lorens A. Imhof for comments and discussions. We gratefully acknowledge support by China Scholarship Council (2009601286, B.W.), the National Natural Science Foundation of China (10972002 and 60736022, L.W.), and the Emmy-Noether program of the Deutsche Forschungsgemeinschaft (C.S.G. and A.T.).

\section{Appendix A: The stationary distribution}

Here, we recall the calculation of the stationary distribution $\psi_{j}$ for a one dimensional birth-death process without absorbing states (Kampen 1997; Gardiner 2004; Claussen and Traulsen 2005). The stationary distribution fulfills the detailed balance condition $\psi_{j-1} T_{j-1}^{+}=\psi_{j} T_{j}^{-}$. We rearrange this to

$$
\psi_{j}=\frac{T_{j-1}^{+}}{T_{j}^{-}} \psi_{j-1}
$$

Therefore

$$
\begin{aligned}
& \psi_{1}=\frac{T_{0}^{+}}{T_{1}^{-}} \psi_{0} \\
& \psi_{2}=\frac{T_{1}^{+}}{T_{2}^{-}} \psi_{1}=\frac{T_{0}^{+} T_{1}^{+}}{T_{2}^{-} T_{1}^{-}} \psi_{0} \\
& \psi_{3}=\frac{T_{2}^{+}}{T_{3}^{-}} \psi_{2}=\frac{T_{0}^{+} T_{1}^{+} T_{2}^{+}}{T_{3}^{-} T_{1}^{-} T_{2}^{-}} \psi_{0} .
\end{aligned}
$$

In general, we have

$$
\psi_{j}=\frac{T_{0}^{+}}{T_{j}^{-}} \prod_{i=1}^{j-1} \frac{T_{i}^{+}}{T_{i}^{-}} \psi_{0}, \quad 1 \leq j \leq N
$$

On the other hand, $\sum_{j=0}^{N} \psi_{j}=1$. Thus, we have

$$
1=\sum_{j=0}^{N} \psi_{j}=\psi_{0}\left(1+\sum_{j=1}^{N} \frac{T_{0}^{+}}{T_{j}^{-}} \prod_{i=1}^{j-1} \frac{T_{i}^{+}}{T_{i}^{-}}\right)
$$

and hence

$$
\psi_{0}=\frac{1}{1+\sum_{j=1}^{N} \frac{T_{0}^{+}}{T_{j}^{-}} \prod_{i=1}^{j-1} \frac{T_{i}^{+}}{T_{i}^{-}}}
$$


Therefore, by Eq. (30)

$$
\psi_{j}=\frac{\frac{T_{0}^{+}}{T_{j}^{-}} \prod_{i=1}^{j-1} \frac{T_{i}^{+}}{T_{i}^{-}}}{1+\sum_{k=1}^{N} \frac{T_{0}^{+}}{T_{k}^{-}} \prod_{i=1}^{k-1} \frac{T_{i}^{+}}{T_{i}^{-}}}, \quad 1 \leq j \leq N .
$$

\section{Appendix B: Estimating the critical mutation rate for the Fermi process}

In this section, we consider the first order term of the Taylor approximation of the stationary distribution for small mutation rates. This provides part of the proof of the Theorem in the main text.

\section{The first order term of the stationary distribution in the mutation rate}

We calculate the first order expansion of the stationary distribution at state 0 under small mutation. Since $T_{N}^{-}=\mu=T_{0}^{+}$, we have

$$
\psi_{0}(\mu)=\frac{1}{1+\mu\left(\sum_{k=1}^{N-1} \frac{1}{T_{k}^{-}} \prod_{i=1}^{k-1} \frac{T_{i}^{+}}{T_{i}^{-}}\right)+\prod_{i=1}^{N-1} \frac{T_{i}^{+}}{T_{i}^{-}}}
$$

Thus, $\left.\frac{d}{d \mu} \psi_{0}\right|_{\mu=0}$ is given by

$$
\left.\frac{d}{d \mu} \psi_{0}\right|_{\mu=0}=-\psi_{0}^{2}(0)[\underbrace{\left.\left(\sum_{k=1}^{N-1} \frac{1}{T_{k}^{-}} \prod_{i=1}^{k-1} \frac{T_{i}^{+}}{T_{i}^{-}}\right)\right|_{\mu=0}}_{C_{1}}+\underbrace{\left.\frac{d}{d \mu}\left(\prod_{i=1}^{N-1} \frac{T_{i}^{+}}{T_{i}^{-}}\right)\right|_{\mu=0}}_{C_{2}}]
$$

This equation is valid for all evolutionary birth-death processes. Substituting Eq. (6) into $C_{1}$ yields

$$
\begin{aligned}
C_{1} & =\sum_{k=1}^{N-1} \frac{N^{2}\{1+\exp [(u k+v) \beta]\}}{k(N-k)} \prod_{i=1}^{k-1} \exp [(u i+v) \beta] \\
& =\sum_{k=1}^{N-1} \frac{N^{2}\{1+\exp [(u k+v) \beta]\}}{k(N-k)} \exp \left[\sum_{i=1}^{k-1}(u i+v) \beta\right] \\
& =\sum_{k=1}^{N-1} \frac{N^{2}\{1+\exp [(u k+v) \beta]\}}{k(N-k)} \exp \left[\left(\frac{u}{2} k+v\right)(k-1) \beta\right]
\end{aligned}
$$


Next, we address $C_{2}$. Let $g(\mu)=\prod_{i=1}^{N-1} \frac{T_{i}^{+}}{T_{i}^{-}}$, therefore $\ln g(\mu)=\sum_{i=1}^{N-1}\left(\ln T_{i}^{+}-\right.$ $\ln {T_{i}}^{-}$). The derivative of this quantity is given by $\frac{d}{d \mu} \ln g(\mu)=\frac{g^{\prime}(\mu)}{g(\mu)}$, which results in $C_{2}=\left.\frac{d}{d \mu} g(\mu)\right|_{\mu=0}=\left.g(0) \frac{d}{d \mu} \ln g(\mu)\right|_{\mu=0}$. On the other hand, $\frac{d}{d \mu} \ln g(\mu)=$ $\sum_{i=1}^{N-1}\left(\frac{T_{i}^{+^{\prime}}}{T_{i}{ }^{+}}-\frac{T_{i}^{-^{\prime}}}{T_{i}^{-}}\right)$. Therefore,

$$
C_{2}=\left.\left.\prod_{i=1}^{N-1}\left(\frac{T_{i}^{+}}{T_{i}^{-}}\right)\right|_{\mu=0} \sum_{i=1}^{N-1}\left(\frac{T_{i}^{+^{\prime}}}{T_{i}^{+}}-\frac{T_{i}^{{\chi^{\prime}}^{\prime}}}{T_{i}^{-}}\right)\right|_{\mu=0} .
$$

By Eq. (6), we have $\left.T_{i}^{+^{\prime}}\right|_{\mu=0}=\frac{N-i}{N}-\left.T_{i}^{+}\right|_{\mu=0}$ and $\left.T_{i}^{-^{\prime}}\right|_{\mu=0}=\frac{i}{N}-\left.T_{i}^{-}\right|_{\mu=0}$. Substituting these expressions into Eq. (38) yields

$$
\begin{aligned}
C_{2}= & \left.\left.\prod_{i=1}^{N-1}\left(\frac{T_{i}^{+}}{T_{i}^{-}}\right)\right|_{\mu=0} \sum_{i=1}^{N-1}\left(\frac{N-i}{N T_{i}^{+}}-\frac{i}{N T_{i}^{-}}\right)\right|_{\mu=0} \\
= & \exp \left[\sum_{i=1}^{N-1}(u i+v) \beta\right] \\
\times & {\left[\sum_{i=1}^{N-1} \frac{N\{1+\exp [-\beta(u i+v)]\}}{i}-\sum_{i=1}^{N-1} \frac{N\{1+\exp [\beta(u i+v)]\}}{N-i}\right] } \\
= & \exp \left[\left(u \frac{(N-1) N}{2}+v(N-1)\right) \beta\right] \\
\times & {\left[\sum_{i=1}^{N-1} \frac{N\{1+\exp [-\beta(u i+v)]\}}{i}-\sum_{i=1}^{N-1} \frac{N\{1+\exp [\beta(u(N-i)+v)]\}}{i}\right] }
\end{aligned}
$$

where we have exchanged the summation variable in the second sum, $i \leftrightarrow N-i$. Next, we can drop common terms in the two sums and arrive at

$$
\begin{aligned}
C_{2}= & N \exp \left[\left(\frac{N-1}{2}(u N+2 v)\right) \beta\right] \exp [-v \beta](1-\exp [(u N+2 v) \beta]) \\
& \times \sum_{i=1}^{N-1} \frac{\exp [-u i \beta]}{i}
\end{aligned}
$$

\section{Scaling of the first order term with $N$}

Next, we estimate the order of $2 C_{1}+C_{2}$. To facilitate the calculation, we classify the $2 \times 2$ games by the payoff difference parameters, $u$ and $v$ 


\begin{tabular}{ll}
\hline \multicolumn{1}{c}{ Classification of the game } \\
\hline Neither $u$ nor $v$ is zero & (i) $u<0$ and $v<0$ \\
& (ii) $u<0$ and $v>0$, coexistence game \\
& (iii) $u<0$ and $v>0$, non-coexistence game \\
& (iv) $u>0$ and $v>0$ \\
& (v) $u>0$ and $v<0$, coordination game \\
& $(v i) u>0$ and $v<0$, non-coordination game \\
& $(v i i) u=0$ and $v<0$ \\
Either $u$ or $v$ is zero & $(v i i i) u=0$ and $v>0$ \\
& $($ ix $u>0$ and $v=0$ \\
& $(x) u<0$ and $v=0$ \\
Both $u$ and $v$ are zero & (xi) $u=0$ and $v=0$
\end{tabular}

With this classification, we have to prove that for case $(i i)$, i.e. the coexistence game, $2 C_{1}+C_{2}$ is greater than $G_{2}(N)$ which is of order $\sqrt{N} \exp [N]$, whereas for all the other cases, $2 C_{1}+C_{2}$ is less than $G_{1}(N)$ which is of order $N \ln N$. We only show the calculations for case $(i)(i i)$ and $(v)$, for the rest of the cases it can be proved by identical techniques. For case $(x i)$ though, it is identical with the case without selection intensity. Further, without loss of generality, we assume that the payoff entries are of order 1. Thus $u$ is of the order of $1 / N$ and $v$ as well as $\lambda=u N+2 v<0$ are of order 1 when $N$ is large. On the other hand, for large $N, \lambda<0$ is equivalent to the risk dominance condition of strategy $B$. Also, since $\beta$ can be absorbed into the payoff entries in the transition probabilities, we let $\beta$ be one for simplicity.

Dominance of strategy $B$ with $u<0$ and $v<0$

For $C_{1}$, we have

$$
\begin{aligned}
C_{1} & =\sum_{i=1}^{N-1} \frac{N^{2}(1+\exp [u i+v])}{i(N-i)} \exp \left[\frac{u}{2}(i-1)^{2}+\left(\frac{u}{2}+v\right)(i-1)\right] \\
& <\sum_{i=1}^{N-1} \frac{2 N^{2}}{i(N-i)} \exp \left[\frac{u}{2}(i-1)^{2}+\left(\frac{u}{2}+v\right)(i-1)\right] \\
& <2 N^{2} \sum_{i=1}^{N-1} \frac{1}{i(N-i)} \\
& =2 N\left(\sum_{i=1}^{N-1} \frac{1}{i}+\sum_{i=1}^{N-1} \frac{1}{N-i}\right) \\
& =4 N H_{N-1}
\end{aligned}
$$

The Harmonic number $H_{N-1}$ is of order $\ln N$ for large $N$, thus $C_{1}$ is smaller than a quantity of order $N \ln N$. 
For $C_{2}$, we have (with $\lambda=u N+2 v<0$ )

$$
\begin{aligned}
C_{2} & =N \exp \left[\frac{N-1}{2} \lambda-v\right](1-\exp [\lambda]) \sum_{k=1}^{N-1} \frac{\exp [-u k]}{k} \\
& \leqslant N \exp \left[\frac{N-1}{2} \lambda-v\right](1-\exp [\lambda]) \exp [-u(N-1)] \sum_{k=1}^{N-1} \frac{1}{k} \\
& =N \exp \left[\frac{N-1}{2} \lambda-v\right](1-\exp [\lambda]) \exp [-u(N-1)] H_{N-1}
\end{aligned}
$$

$u<0$ is of order $1 / N$ and $\lambda<0$ is of order 1 . Thus, $N \exp \left[\frac{N-1}{2} \lambda-v\right](1-\exp [\lambda])$ $\exp [-u(N-1)] H_{N-1}$ is of order $N \ln N \exp [-N]$, which is much smaller than $N \ln N$. Thus, $C_{2}$ can be neglected compared to $C_{1} ; G_{1}(N)=8 N H_{N-1}>2 C_{1}+C_{2}$ scales at most with $N \ln N$.

Coordination game with $u>0$ and $v<0$

To estimate the order of $C_{1}$, let

$$
F(i)=\frac{u}{2} i^{2}+\left(\frac{u}{2}+v\right) i
$$

We have $F(0)=0$ and $F(N-1)=(N-1)(u N+2 v) / 2=(N-1) \lambda / 2<0$. On the other hand, $F^{\prime \prime}(i)=\frac{u}{2}$. Since $u>0, F(i)$ is a convex function which implies

$$
\begin{aligned}
F(i) & =F\left(\frac{i}{N-1}(N-1)+\left(1-\frac{i}{N-1}\right) 0\right) \\
& \leq \frac{i}{N-1} F(N-1)+\left(1-\frac{i}{N-1}\right) F(0) \\
& \leq 0
\end{aligned}
$$

where equality holds for $i=0$ only. Therefore for $C_{1}$, we have

$$
\begin{aligned}
C_{1} & =\sum_{i=1}^{N-1} \frac{N^{2}(1+\exp [u i+v])}{i(N-i)} \exp \underbrace{\left[\frac{u}{2}(i-1)^{2}+\left(\frac{u}{2}+v\right)(i-1)\right]}_{F(i-1)} \\
& <\sum_{i=1}^{N-1} \frac{N^{2}(1+\exp [u i+v])}{i(N-i)} \\
& <\sum_{i=1}^{N-1} \frac{N^{2}(1+\exp [u(N-1)+v])}{i(N-i)}
\end{aligned}
$$




$$
\begin{aligned}
& =N(1+\exp [u(N-1)+v])\left(\sum_{i=1}^{N-1} \frac{1}{i}+\sum_{i=1}^{N-1} \frac{1}{N-i}\right) \\
& =2(1+\exp [u(N-1)+v]) N H_{N-1}
\end{aligned}
$$

Considering that $u$ and $v$ are of order $1 / N$ and $1 . C_{1}$ is less than a quantity of order $N \ln N$.

For $C_{2}$, since $u>0$, we have

$$
\begin{aligned}
C_{2} & =N \exp \left[\frac{N-1}{2} \lambda-v\right](1-\exp [\lambda]) \sum_{k=1}^{N-1} \frac{\exp [-u k]}{k} \\
& \leqslant N \exp \left[\frac{N-1}{2} \lambda-v\right](1-\exp [\lambda]) \exp [-u] \sum_{k=1}^{N-1} \frac{1}{k} \\
& =N \exp \left[\frac{N-1}{2} \lambda-v\right](1-\exp [\lambda]) \exp [-u] H_{N-1}
\end{aligned}
$$

In analogy to the order analysis for Eq. (43), $C_{2}$ is much smaller than $C_{1}$. Hence, $2 C_{1}+C_{2}$ scales with $N$ as $N \ln N$. Thus our quantity $G_{1}(N)$ in the proof is $4(1+$ $\exp [u(N-1)+v]) N H_{N-1}$.

Coexistence of strategy $A$ and $B$ with $u<0$ and $v>0$

We show that for a coexistence game, $2 C_{1}+C_{2}$ is greater than a quantity of order $\sqrt{N} \exp (N)$. For $C_{1}$, we have

$$
\begin{aligned}
C_{1} & =\sum_{i=1}^{N-1} \frac{N^{2}(1+\exp [u i+v])}{i(N-i)} \exp \left[\frac{u}{2}(i-1)^{2}+\left(\frac{u}{2}+v\right)(i-1)\right] \\
& >4 \sum_{i=1}^{N-1} \exp [u i+v] \exp \left[\frac{u}{2}(i-1)^{2}+\left(\frac{u}{2}+v\right)(i-1)\right] \\
& =4 \sum_{i=1}^{N-1} \exp \left[\frac{u}{2} i^{2}+\left(\frac{u}{2}+v\right) i\right] \\
& =4 \exp \left[-\frac{u}{2}\left(\frac{1}{2}+\frac{v}{u}\right)^{2}\right] \sum_{i=1}^{N-1} \exp \left[\frac{u}{2}\left(i+\frac{1}{2}+\frac{v}{u}\right)^{2}\right]
\end{aligned}
$$

When the population size $N$ is large, we can set $x=i /(N-1)$ and approximate the sum in the above equation by an integral,

$$
(N-1) \int_{0}^{1} \exp \left[-\frac{1}{2}\left(\sqrt{-u}\left((N-1) x+\frac{1}{2}+\frac{v}{u}\right)\right)^{2}\right] d x
$$


Let $t=\sqrt{-u}\left[(N-1) x+\frac{1}{2}+\frac{v}{u}\right]$, then the above integral is

$$
\frac{1}{N-1} \sqrt{-\frac{2 \pi}{u}}\left[\Phi\left(\sqrt{-u}\left(N-\frac{1}{2}+\frac{v}{u}\right)\right)-\Phi\left(\sqrt{-u}\left(\frac{1}{2}+\frac{v}{u}\right)\right)\right]
$$

where $\Phi(x)=\frac{1}{\sqrt{2 \pi}} \int_{-\infty}^{x} e^{-t^{2} / 2} d t$ is the cumulative distribution function of the Gaussian distribution. For a coexistence game, $u i+v=0$ has a solution $i$ between 1 and $N-1$. Thus $-\frac{v}{u} \leq N-1$. With this, we have $0<N-\frac{1}{2}+\frac{v}{u}$. Hence, $\sqrt{-u}\left(N-\frac{1}{2}+\frac{v}{u}\right)$ is of order $+\sqrt{N}$ and approaches $+\infty$ as the population size $N$ goes to infinity. Thus, $\Phi\left(\sqrt{-u}\left(N-\frac{1}{2}+\frac{v}{u}\right)\right)$ approaches 1 as $N$ approaches infinity. Similarly, a coexistence game implies $0<-\frac{v}{u}$ and thus $\sqrt{-u}\left(\frac{1}{2}+\frac{v}{u}\right)$ scales as $-\sqrt{N}$. Therefore, the second term $\Phi\left(\sqrt{-u}\left(\frac{1}{2}+\frac{v}{u}\right)\right)$ approaches 0 as $N$ approaches infinity. This means that the sum in Eq. (50) is larger than $\sqrt{-\frac{2 \pi}{u}}$ for large $N$, yielding a lower bound for $C_{1}$,

$$
C_{1}>4 \sqrt{-\frac{2 \pi}{u}} \exp \left[-\frac{u}{2}\left(\frac{1}{2}+\frac{v}{u}\right)^{2}\right]
$$

Now, $u<0$ scales as $1 / N$, whereas $v$ becomes independent of $N$ for large $N$. Hence, $C_{1}$ scales as $\sqrt{N} \exp [N]$, i.e. it increases more than exponentially with $N$. For $C_{2}$, the order estimation is identical to Eq. (43), $C_{2}$ becomes infinitely small for large $N$. Therefore, $2 C_{1}+C_{2}$ scales as $\sqrt{N} \exp [N]$ and the mutation rate has to go to zero rapidly to ensure that the approximation remains good when the population size is increased. Thus $G_{2}(N)$ is $8 \sqrt{-\frac{2 \pi}{u}} \exp \left[-\frac{u}{2}\left(\frac{1}{2}+\frac{v}{u}\right)^{2}\right]$.

\section{A numerically accessible bound for the mutation rate}

In this part of the Appendix, we show, for a given non-coexistence game, how the critical mutation rate depends on the payoff entries. By the proof provided above, this mutation rate is $\left(2 C_{1}\right)^{-1} \varepsilon$ for large population size, where $\varepsilon$ is the given tolerance of the error. Thus we only need to derive the relationship between $C_{1}$ and the payoff entries.

For coordination games, Eq. (47) provides such a relationship. For dominance games, however, it is not straightforward from Eq. (42). But based on Expression (42), we have

$$
C_{1}<\sum_{i=1}^{N-1} \frac{2 N^{2}}{i(N-i)} \exp \left[\frac{u}{2}(i-1)^{2}+\left(\frac{u}{2}+v\right)(i-1)\right]
$$


By the Cauchy-Schwarz inequality,

$$
C_{1}<\left(\sum_{i=1}^{N-1} \frac{2 N^{2}}{i(N-i)}\right)^{\frac{1}{2}}(\sum_{i=1}^{N-1} \underbrace{\exp \left[\frac{u}{2}(i-1)^{2}+\left(\frac{u}{2}+v\right)(i-1)\right]}_{R(i-1)})^{\frac{1}{2}}
$$

By using $\sum_{i=1}^{N-1} R(i-1)=\sum_{i=0}^{N-2} R(i)=\sum_{i=1}^{N-1} R(i)+R(0)-R(N-1)$, the above inequality can be rewritten as

$$
\begin{gathered}
C_{1}<\left(\sum_{i=1}^{N-1} \frac{2 N^{2}}{i(N-i)}\right)^{\frac{1}{2}}\left(\sum_{i=1}^{N-1} \exp \left[\frac{u}{2} i^{2}+\left(\frac{u}{2}+v\right) i\right]+1\right. \\
\left.-\exp \left[(N-1) \frac{N u+2 v}{2}\right]\right)^{\frac{1}{2}}
\end{gathered}
$$

The first factor of the r.h.s of the inequality, by Eq. (27), scales as $2 \sqrt{N \ln N}$. The second factor is similar to the expressions obtained by Eqs. (49), (50), (51), (52). It can be approximated by the square root of $\exp \left[-\frac{u}{2}\left(\frac{1}{2}+\frac{v}{u}\right)^{2}\right] \sqrt{-\frac{2 \pi}{u}}[\Phi(\sqrt{-u}(N-$ $\left.\left.\left.\frac{1}{2}+\frac{v}{u}\right)\right)-\Phi\left(\sqrt{-u}\left(\frac{1}{2}+\frac{v}{u}\right)\right)+1\right]$ for large $N$ where $\Phi(x)$ is the standard Gaussian distribution function. Thus

$$
\begin{aligned}
C_{1}<2 & \sqrt{N \ln N} \sqrt[4]{-\frac{2 \pi}{u}} \exp \left[-\frac{u}{4}\left(\frac{1}{2}+\frac{v}{u}\right)^{2}\right] \\
& \times \sqrt{\Phi\left(\sqrt{-u}\left(N-\frac{1}{2}+\frac{v}{u}\right)\right)-\Phi\left(\sqrt{-u}\left(\frac{1}{2}+\frac{v}{u}\right)\right)+1}
\end{aligned}
$$

This allows us to estimate a numerical value for the critical mutation bound for given payoff entries of a non-coexistence game and error tolerance without the need to evaluate sums. If the system is not too large such that sums can be evaluated numerically, Eq. (46) gives a more precise estimate.

\section{Appendix C: Estimating the critical mutation rate for general imitation processes}

For the general imitation process with mutations, an individual is picked up from the well mixed population of size $N$. With probability $1-\mu$, imitation occurs: The focal individual imitates another random individual with a probability $g\left(\beta \Delta \pi_{i}\right)$, where $\Delta \pi_{i}=\pi_{A}-\pi_{B}$ and $\beta$ is the selection intensity. Here $g(x)$ is an increasing function. This implies that the more successful the opponent is, the more likely the focal individual imitates it. With probability $\mu<1 / 2$, mutation or exploration occurs: The focal individual switches to the opposite strategy. 
In analogy to the transition probabilities given by Eq. (6), we have

$$
\begin{aligned}
T_{i}^{+} & =(1-\mu) \frac{i}{N} \frac{N-i}{N} g\left(+\beta \Delta \pi_{i}\right)+\mu \frac{N-i}{N} \\
T_{i}^{-} & =(1-\mu) \frac{i}{N} \frac{N-i}{N} g\left(-\beta \Delta \pi_{i}\right)+\mu \frac{i}{N} .
\end{aligned}
$$

and $1-T_{i}^{+}-T_{i}^{-}$. In this Appendix, we show that the Theorem is also valid for a wide class of imitation processes. The only technical requirement is that the imitation function is strictly increasing and that its derivative is continuous.

\section{The form of the first order term}

For the general imitation process with mutations, we still have $C_{1}$ and $C_{2}$ defined in Eqs. (16) and (18). For $C_{1}$, we obtain

$$
\begin{aligned}
C_{1} & =\left.\left(\sum_{i=1}^{N-1} \frac{1}{T_{i}^{-}} \prod_{k=1}^{i-1} \frac{T_{k}^{+}}{T_{k}^{-}}\right)\right|_{\mu=0} \\
& =\sum_{i=1}^{N-1} \frac{N^{2}}{i(N-i)} \frac{1}{g\left(-\beta \Delta \pi_{i}\right)} \prod_{k=1}^{i-1} \frac{g\left(+\beta \Delta \pi_{k}\right)}{g\left(-\beta \Delta \pi_{k}\right)}
\end{aligned}
$$

By making use of the identity $x=\exp [\ln x]$ for $x=\prod_{k=1}^{i-1} \frac{g\left(+\beta \Delta \pi_{k}\right)}{g\left(-\beta \Delta \pi_{k}\right)}$, we arrive at

$$
C_{1}=\sum_{i=1}^{N-1} \frac{N^{2}}{i(N-i)} \frac{1}{g\left(-\beta \Delta \pi_{i}\right)} \exp \left[\sum_{k=1}^{i-1} \ln \left[\frac{g\left(+\beta \Delta \pi_{k}\right)}{g\left(-\beta \Delta \pi_{k}\right)}\right]\right]
$$

For $C_{2}$, note that the derivation of Eq. (39) is independent of the imitation function given, thus it is valid for all imitation processes. We have

$$
\begin{aligned}
C_{2} & =\left.\left.\prod_{k=1}^{N-1}\left(\frac{T_{k}^{+}}{T_{k}^{-}}\right)\right|_{\mu=0} \sum_{k=1}^{N-1}\left(\frac{N-k}{N T_{k}+}-\frac{k}{N T_{k}-}\right)\right|_{\mu=0} \\
& =\prod_{k=1}^{N-1} \frac{g\left(+\beta \Delta \pi_{k}\right)}{g\left(-\beta \Delta \pi_{k}\right)} \sum_{k=1}^{N-1} \frac{N}{N-k}\left(\frac{1}{g\left(+\beta \Delta \pi_{N-k}\right)}-\frac{1}{g\left(-\beta \Delta \pi_{k}\right)}\right) \\
& =\exp \left[\sum_{k=1}^{N-1} \ln \left[\frac{g\left(+\beta \Delta \pi_{k}\right)}{g\left(-\beta \Delta \pi_{k}\right)}\right]\right] \sum_{k=1}^{N-1} \frac{N}{N-k}\left(\frac{1}{g\left(+\beta \Delta \pi_{N-k}\right)}-\frac{1}{g\left(-\beta \Delta \pi_{k}\right)}\right)
\end{aligned}
$$

For $C_{2}$, if $\frac{1}{g\left(+\beta \Delta \pi_{N-k}\right)}-\frac{1}{g\left(-\beta \Delta \pi_{k}\right)}$ is non-negative for all the $k$, then $C_{2}$ is non-negative. Since $g(x)$ is an increasing function, this is equivalent to $\Delta \pi_{N-k} \leq-\Delta \pi_{k}$, i.e., $u N+2 v \leq 0$. If this is not the case, we can exchange strategy $A$ and $B$, as described 
in the main text. This yields a transformed game which fulfills $\tilde{u} N+2 \tilde{v} \leq 0$ without influencing the main results. Therefore, we always consider the case for $u N+2 v \leq 0$, such that both $C_{1}$ and $C_{2}$ are non-negative.

\section{Scaling of the first order term with $N$}

To estimate the order of $2 C_{1}+C_{2}$, we absorb the selection intensity $\beta$ into the payoff difference term in analogy to the proof above, i.e. we formally set $\beta=1$. The quantity $u$ is of order $1 / N$ and $v$ is of order 1 . Without loss of generality (see above), $u N+2 v \leq 0$ is also assumed to ensure $C_{2}>0$.

For the coordination game, $u>0$ and $v<0$, we only need to prove that $2 C_{1}+C_{2}$ is less than a quantity of order $N \ln N$. For $C_{1}$, we have

$$
\begin{aligned}
C_{1} & =\sum_{i=1}^{N-1} \frac{N^{2}}{i(N-i)} \frac{1}{g\left(-\Delta \pi_{i}\right)} \exp \left[\sum_{k=1}^{i-1} \ln \left[\frac{g\left(\Delta \pi_{k}\right)}{g\left(-\Delta \pi_{k}\right)}\right]\right] \\
& <\sum_{i=1}^{N-1} \frac{N^{2}}{i(N-i)} \frac{1}{g\left(-\Delta \pi_{N}\right)} \exp \left[\sum_{k=1}^{i-1} \ln \left[\frac{g\left(\Delta \pi_{k}\right)}{g\left(-\Delta \pi_{k}\right)}\right]\right]
\end{aligned}
$$

By Lagrange mean value theorem, for every $1 \leq k \leq N-1$ there exists $\xi_{k} \in[0,1]$, s.t.

$$
\begin{aligned}
\ln \left[g\left(\Delta \pi_{k}\right)\right]-\ln \left[g\left(-\Delta \pi_{k}\right)\right] & =u N \frac{g^{\prime}\left(u N \xi_{k}+v\right)}{g\left(u N \xi_{k}+v\right)}\left(\Delta \pi_{k}-\left(-\Delta \pi_{k}\right)\right) \\
& \leq 2 u N \frac{M}{g(v)} \Delta \pi_{k}
\end{aligned}
$$

where $M$ is the maximum of $g^{\prime}(x)$ for $x \in[v, u N+v]$. Since $v$ and $u N+v$ are of order $1, M$ only depends on the imitation function and payoff entries rather than the population size $N$ for large $N$. Thus we can consider it to be of order 1 in what concerns the scaling of $N$. On the other hand, since $g^{\prime}(x)$ is continuous as we assume, there exists $y^{*} \in[0,1]$ such that $M=g^{\prime}\left(y^{*}\right)>0$. Therefore, $u N \frac{M}{g(v)}>0$ becomes independent of $N$ for large $N$. This implies

$$
C_{1}<\sum_{i=1}^{N-1} \frac{N^{2}}{i(N-i)} \frac{1}{g\left(-\Delta \pi_{N}\right)} \exp \left[2 u N \frac{M}{g(v)} \sum_{k=1}^{i-1} \Delta \pi_{k}\right]
$$

Therefore, it degenerates to Eq. (46) for coordination game of the Fermi process. Following the proof therein, finally we arrived at

$$
C_{1}<2 \frac{1}{g\left(-\Delta \pi_{N}\right)} N H_{N-1}
$$


Since $g\left(-\Delta \pi_{N}\right)=g(-u N-v)$ is only dependent on the imitation function and the payoff entries, it is independent of $N$. Thus, $C_{1}$ is smaller than a quantity of order $N \ln N$.

Next, we consider $C_{2}$. We have

$$
C_{2}=\exp \underbrace{\left[\sum_{k=1}^{N-1} \ln \left[\frac{g\left(\Delta \pi_{k}\right)}{g\left(-\Delta \pi_{k}\right)}\right]\right]}_{D_{1}} \underbrace{\sum_{k=1}^{N-1} \frac{N}{N-k}\left(\frac{1}{g\left(\Delta \pi_{N-k}\right)}-\frac{1}{g\left(-\Delta \pi_{k}\right)}\right)}_{D_{2}},
$$

which is a product of $\exp \left[D_{1}\right]$ and $D_{2}$. For $D_{1}$, we have

$$
\begin{aligned}
D_{1} & =\sum_{k=1}^{N-1} \ln \left[\frac{g\left(\Delta \pi_{k}\right)}{g\left(-\Delta \pi_{k}\right)}\right] \\
& =\sum_{k=1}^{N-1} \ln \left[g\left(\Delta \pi_{k}\right)\right]-\sum_{k=1}^{N-1} \ln \left[g\left(-\Delta \pi_{k}\right)\right] \\
& =\sum_{k=1}^{N-1} \ln \left[g\left(\Delta \pi_{k}\right)\right]-\sum_{k=1}^{N-1} \ln \left[g\left(-\Delta \pi_{N-k}\right)\right] \\
& =\sum_{k=1}^{N-1}\left(\ln \left[g\left(\Delta \pi_{k}\right)\right]-\ln \left[g\left(-\Delta \pi_{N-k}\right)\right]\right)
\end{aligned}
$$

Again, by Lagrange mean value theorem, for every $1 \leq k \leq N-1$, there exists $\zeta_{k} \in[0,1]$, s.t.

$$
\begin{aligned}
\ln \left[g\left(\Delta \pi_{k}\right)\right]-\ln \left[g\left(-\Delta \pi_{N-k}\right)\right] & =u N \frac{g^{\prime}\left(u N \zeta_{k}+v\right)}{g\left(u N \zeta_{k}+v\right)}\left(\Delta \pi_{k}-\left(-\Delta \pi_{N-k}\right)\right) \\
& \leq u N \frac{M}{g(v)}(u N+2 v)
\end{aligned}
$$

where $M>0$ is the maximum of $g^{\prime}(x)$ on $[v, u N+v]$ as defined above. Thus we have

$$
\sum_{k=1}^{N-1} \ln \left[\frac{g\left(\Delta \pi_{k}\right)}{g\left(-\Delta \pi_{k}\right)}\right]<u(N-1) N \frac{M}{g(v)}(u N+2 v)
$$

Remembering that $u N+2 v$ is negative and of order $1, u(N-1) N \frac{M}{g(v)}(u N+2 v)$ is smaller than zero and of order $N$ for large $N$. Therefore, exp $\left[\sum_{k=1}^{N-1} \ln \left[\frac{g\left(\Delta \pi_{k}\right)}{g\left(-\Delta \pi_{k}\right)}\right]\right]$ is smaller that a quantity of order $\exp [-N]$. 
For $D_{2}$, since $u>0, \Delta \pi_{k}$ is increasing with $k$. In addition, $g(x)$ is increasing, we have

$$
\begin{aligned}
\frac{1}{g\left(+\Delta \pi_{N-k}\right)}-\frac{1}{g\left(-\Delta \pi_{k}\right)} & =\frac{g\left(-\Delta \pi_{k}\right)-g\left(+\Delta \pi_{N-k}\right)}{g\left(-\Delta \pi_{k}\right) g\left(+\Delta \pi_{N-k}\right)} \\
& <\frac{g\left(-\Delta \pi_{k}\right)-g\left(+\Delta \pi_{N-k}\right)}{g\left(-\Delta \pi_{N}\right) g\left(+\Delta \pi_{0}\right)}
\end{aligned}
$$

By Lagrange mean value theorem, there exists $\eta_{k} \in[0,1]$ s.t.

$$
\begin{aligned}
g\left(-\Delta \pi_{k}\right)-g\left(\Delta \pi_{N-k}\right) & =g^{\prime}\left(-\Delta \pi_{k}+\eta_{k}\left(-\Delta \pi_{k}-\Delta \pi_{N-k}\right)\right)\left(-\Delta \pi_{k}-\Delta \pi_{N-k}\right) \\
& <-H(u N+2 v)
\end{aligned}
$$

where $H>0$ is the maximum of $g^{\prime}(x)$ on $[-v,+v]$, where $\Delta \pi_{k}$ and $-\Delta \pi_{k}$ lie. In analogy to previous discussion, it is independent of $N$ when $N$ is large. Thus we have

$$
\frac{1}{g\left(+\Delta \pi_{N-k}\right)}-\frac{1}{g\left(-\Delta \pi_{k}\right)}<\frac{-H(u N+2 v)}{g(-u N-v) g(v)}
$$

Further, we have

$$
\begin{aligned}
\sum_{k=1}^{N-1} \frac{N}{N-k}\left(\frac{1}{g\left(+\Delta \pi_{N-k}\right)}-\frac{1}{g\left(-\Delta \pi_{k}\right)}\right) & <\left(\frac{-H(u N+2 v)}{g(-u N-v) g(v)}\right) \sum_{k=1}^{N-1} \frac{N}{N-k} \\
& =\left(\frac{-H(u N+2 v)}{g(-u N-v) g(v)}\right) N H_{N-1}
\end{aligned}
$$

Note that $\frac{-H(u N+2 v)}{g(-u N-v) g(v)}$ positive and independent of $N$ for large $N . D_{2}$ is smaller than a quantity of $\operatorname{order} N \ln N$. Finally, $C_{2}=\exp \left[D_{1}\right] D_{2}$ is of order $N \ln N \exp [-N]$; it becomes infinitely small for large $N$. This means that the scaling of $2 C_{1}+C_{2}$ is determined by the scaling of $C_{1}$ and thus the critical mutation rate scales as $N \ln N$.

For the coexistence game and dominance game, the procedure of the proof for general imitation function is also identical to that of the coordination game: For $C_{1}$, we make use of Lagrange mean value theorem to establish a relationship between $\ln \left[\frac{g\left(\Delta \pi_{k}\right)}{g\left(-\Delta \pi_{k}\right)}\right]$ and $\Delta \pi_{k}$, then it can be deduced by the proof the corresponding game for the Fermi process. For $C_{2}$, for all games, it is infinitely small for large population size. The proof is identical with that of the coordination game for general imitation function.

\section{References}

Antal T, Scheuring I (2006) Fixation of strategies for an evolutionary game in finite populations. Bull Math Biol 68:1923-1944

Antal T, Nowak MA, Traulsen A (2009) Strategy abundance in $2 \times 2$ games for arbitrary mutation rates. J Theor Biol 257:340-344 
Antal T, Ohtsuki H, Wakeley J, Taylor PD, Nowak MA (2009) Evolution of cooperation by phenotypic similarity. Proc Natl Acad Sci USA 106:8597-8600

Antal T, Traulsen A, Ohtsuki H, Tarnita CE, Nowak MA (2009) Mutation-selection equilibrium in games with multiple strategies. J Theor Biol 258:614-622

Blume LE (1993) The statistical mechanics of strategic interaction. Games Econ Behav 5:387-424

Brémaud P (1999) Markov chains: Gibbs fields, Monte Carlo simulation, and queues. Springer, Berlin

Bürger R (2000) The mathematical theory of selection, recombination, and mutation. Wiley, New York

Chalub FA, Souza MO (2009) From discrete to continuous evolution models: A unifying approach to driftdiffusion and replicator dynamics. Theor Popul Biol 76:268-277

Claussen JC, Traulsen A (2005) Non-Gaussian fluctuations arising from finite populations: exact results for the evolutionary Moran process. Phys Rev E 71:025101(R)

Cressman R (1992) The stability concept of evolutionary game theory. Lecture Notes in Biomathematics, vol 94. Springer, Berlin

Crow JF, Kimura M (1970) An introduction to population genetics theory. Harper and Row, New York

Durrett R (1996) Probability: theory and examples. Citeseer

Ewens WJ (2004) Mathematical population genetics. Springer, New York

Foster D, Young P (1990) Stochastic evolutionary game dynamics. Theor Popul Biol 38:219-232

Fudenberg D, Harris C (1992) Evolutionary dynamics with aggregate shocks. J Econ Theory 57:420-441

Fudenberg D, Imhof LA (2006) Imitation process with small mutations. J Econ Theory 131:251-262

Fudenberg D, Imhof LA (2008) Monotone imitation dynamics in large populations. J Econ Theory 140: 229-245

Gardiner CW (2004) Handbook of Stochastic Methods, 3rd edn. Springer, New York

Goel N, Richter-Dyn N (1974) Stochastic models in biology. Academic Press, New York

Hauert C, Traulsen A, Brandt H, Nowak MA, Sigmund K (2007) Via freedom to coercion: the emergence of costly punishment. Science 316:1905-1907

Hofbauer J, Sigmund K (1998) Evolutionary games and population dynamics. Cambridge University Press, Cambridge

Imhof LA, Nowak MA (2006) Evolutionary game dynamics in a Wright Fisher process. J Math Biol 52: $667-681$

Imhof LA, Fudenberg D, Nowak MA (2005) Evolutionary cycles of cooperation and defection. Proc Natl Acad Sci USA 102:10797-10800

Kallenberg O (2002) Foundations of modern probability. Springer, Berlin

Kampen NGv (1997) Stochastic processes in physics and chemistry, 2nd edn. Elsevier, Amsterdam

Kandori M, Mailath GJ, Rob R (1993) Learning, mutation, and long run equilibria in games. Econometrica 61:29-56

Karlin S, Taylor HMA (1975) A first course in stochastic processes, 2nd edn. Academic, London

Levin DA, Peres Y, Wilmer EL (2009) Markov chains and mixing times. American Mathematical Society, Providence

Nowak MA (2006) Evolutionary dynamics. Harvard University Press, Cambridge

Nowak MA, Sasaki A, Taylor C, Fudenberg D (2004) Emergence of cooperation and evolutionary stability in finite populations. Nature 428:646-650

Ohtsuki H, Hauert C, Lieberman E, Nowak MA (2006) A simple rule for the evolution of cooperation on graphs. Nature 441:502-505

Roca CP, Cuesta JA, Sanchez A (2009) Evolutionary game theory: temporal and spatial effects beyond replicator dynamics. Phys Life Rev 6:208-249

Santos FC, Pacheco JM (2005) Scale-free networks provide a unifying framework for the emergence of cooperation. Phys Rev Lett 95:098104

Sella G, Hirsh AE (2005) The application of statistical physics to evolutionary biology. Proc Natl Acad Sci USA 102(27):9541-9546

Sigmund K, DeSilva H, Traulsen A, Hauert C (2010) Social learning promotes institutions for governing the commons. Nature 466:861-863

Szabó G, Fáth G (2007) Evolutionary games on graphs. Phys Rep 446:97-216

Szabó G, Tőke C (1998) Evolutionary Prisoner's Dilemma game on a square lattice. Phys Rev E 58:69

Tarnita CE, Ohtsuki H, Antal T, Fu F, Nowak MA (2009) Strategy selection in structured populations. J Theor Biol 259:570-581

Taylor C, Fudenberg D, Sasaki A, Nowak MA (2004) Evolutionary game dynamics in finite populations. Bull Math Biol 66:1621-1644 
Traulsen A, Nowak MA (2007) Chromodynamics of cooperation in finite populations. PLoS One 2:e270

Traulsen A, Nowak MA, Pacheco JM (2006) Stochastic dynamics of invasion and fixation. Phys Rev E 74:011909

Traulsen A, Shoresh N, Nowak MA (2008) Analytical results for individual and group selection of any intensity. Bull Math Biol 70:1410-1424

Traulsen A, Hauert C, De Silva H, Nowak MA, Sigmund K (2009) Exploration dynamics in evolutionary games. Proc Natl Acad Sci USA 106:709-712

Van Segbroeck S, Santos FC, Lenaerts T, Pacheco JM (2009) Reacting differently to adverse ties promotes cooperation in social networks. Phys Rev Lett 102:058105

van Veelen M (2007) Hamilton's missing link. J Theor Biol 246:551-554

Wang J, Wu B, Chen X, Wang L (2010) Evolutionary dynamics of public goods games with diverse contributions in finite populations. Phys Rev E 81:056103

Wu B, Altrock PM, Wang L, Traulsen A (2010) Universality of weak selection. Phys Rev E 82:046106 